EGU21-16055, updated on 28 Jan 2022

https://doi.org/10.5194/egusphere-egu21-16055

EGU General Assembly 2021

(c) Author(s) 2022. This work is distributed under

the Creative Commons Attribution 4.0 License.

\title{
Geological context of recent Lunar landing sites using Multispectral analysis.
}

\author{
Jourdain Mcilquham, Anouk M Borst, Elyse J Allender, and Bernard Foing \\ University of St Andrews
}

Geological context of recent lunar landing sites using multispectral analysis. (Mcilquham J, Borst A, Allender E and Foing B)

The Moon Mineralogy Mapper (M3) was a guest instrument aboard the Chandrayaan-1 mission. The instrument collected spectral data, ranging from $430 \mathrm{~nm}$ to $3000 \mathrm{~nm}$ at an average resolution of $140 \mathrm{~m} /$ pixel. This research utilises M3 spectral data to visualise and understand the geology of lunar landing sites visited by Chang'e 4 and 5. The aims of this study are aligned to lunar exploration goals produced by the National Research Council. We use Python scripts to undertake data analysis, creating site maps using continuum removal methods and assigning RGB image channels to highlight absorption features of interest. The Chang'e 4 landing site is located on the lunar far side within the Von Karman crater, located in the large South Pole Aitken impact basin. At Von Karman lunar mantle or lower crustal material may be exposed in the central peak. This could provide valuable insights into lunar geological history. We create maps to visualise the location of pyroxene end-members and olivine-rich rocks of the Von Karman crater, adding data to understand the composition of the deeper lunar lithologies. Orbital data presented in this study can be compared with ground-truth data gathered from the Yutu 2 rover to confirm the minerals present. More recently the Chang'e 5 mission provided a further landing site for study. Using the same methods as presented above we will compare its spectral composition to the Chang'e 4 landing site. Our maps can help to understand the key factors used to determine a suitable landing site and potentially a suitable location for a lunar base. By comparing Chang'e landing sites this study provides a unique insight into the craters in which they landed, allowing direct comparisons to be drawn. Preliminary findings identify non-mare units within the Von Karman crater as well as various Ca-rich and Ca-poor pyroxene-bearing lithologies. 